\title{
Ultrasound-assisted surgical retrieval of the lost femoral trial head during total hip arthroplasty
}

\author{
Kuiran Jiao, MD (D) Abistanand Ankam, MD, FRCA
}

Received: 15 July 2019/Revised: 24 July 2019/Accepted: 31 July 2019/Published online: 13 August 2019

(C) The Author(s) 2019

\section{To the Editor,}

Currently, approximately 400,000 total hip replacement (THR) surgeries are performed per year in the United States, with numbers expected to increase. ${ }^{1,2}$ During this joint replacement procedure, a "trial femoral head"-a temporary implant similar to a permanent implant-is usually sited to test the tightness and mobility of the hip in a variety of positions prior to the implantation of a permanent femoral head. A rare complication, dislodgment of the trial femoral head from the stem, may occur and can become lost in the surgical field. Since the trial femoral head is made of plastic produced from an inert radiolucent polyphenylsulfone, it may be challenging to locate it using standard intraoperative fluoroscopy. We report a case in which the trial head was successfully identified and retrieved with the assistance of an ultrasound scan.

A 65-yr-old man $(200 \mathrm{~cm}, 107 \mathrm{~kg}$, body mass index 26.7 $\mathrm{kg} \cdot \mathrm{m}^{-2}$ ) with a history of hypertension and atrial fibrillation underwent a primary left THR (spinal anesthesia, propofol intravenous infusion for sedation). During the procedure, the trial femoral head (Biomet, 36 $\mathrm{mm}$ ) was unexpectedly dislodged and lost in the surgical field. It wasn't possible to identify the radiolucent femoral head using intraoperative fluoroscopy despite multiple attempts. We suggested the use of an ultrasound scan to locate the trial head. A GE Logiq E ultrasound machine (GE Medical Systems Co., Ltd, Jiangsu, China) with a "C 1-5 curvilinear probe" was employed to scan the left lower quadrant of the anterior pelvic area. During the scan, a spherical contoured foreign body was quickly identified 3

\footnotetext{
K. Jiao, MD (ه) · A. Ankam, MD, FRCA

Department of Anesthesiology, Guthrie Robert Packer Hospital, Sayre, PA, USA

e-mail: kuiran.jiao@guthrie.org
}

$\mathrm{cm}$ deep from the skin within the iliopsoas muscles in both the transverse and sagittal plane. Multiple subsequent scanning planes showed that this structure was solitary and not connected to any other known bony structure, indicating the trial femoral head (Figure). A separate incision was made along the iliac crest and the trial head was successfully retrieved from within the iliacus muscle. The operation then proceeded uneventfully, the postoperative period was uneventful, and the patient was discharged on the fourth day postoperative day.

There are several options when challenged with such a situation: leave the trial head in the body without further intervention; extend the hip incision to retrieve it during the arthroplasty procedure; or complete the arthroplasty procedure, determine the location of the trial femoral head using advanced imaging techniques (such as computed tomography and magnetic resonance imaging), and arrange for a subsequent surgical intervention. ${ }^{3}$ Typically, the third option is chosen but this is time-consuming and involves coordinating multidisciplinary teams. Furthermore, transportation of an anesthetized patient out of the operating room for advanced imaging is challenging in itself and it may compromise the patients' safety. Finally, it causes additional anxiety, frustration, and dissatisfaction for the patient and family.

Most case reports indicate that, after being lost in the surgical field, the trial heads had migrated to adjacent cavities, beneath or along the iliopsoas muscle through the lacuna musculorum of the inguinal canal along the anterior pelvic brim. ${ }^{3}$ In this region, other than the ileum, there are no bony structures. Theoretically, a hyperechoic structure should be readily identified in this area with ultrasound scanning. We are unaware of another report that describes the use of the ultrasound to locate a lost trial femoral head 
Figure Intraoperative ultrasound image with probe in the sagittal plane showing the curvilinear hyperechoic shadow (arrows) represented by a trial femoral head lost during hip arthroplasty

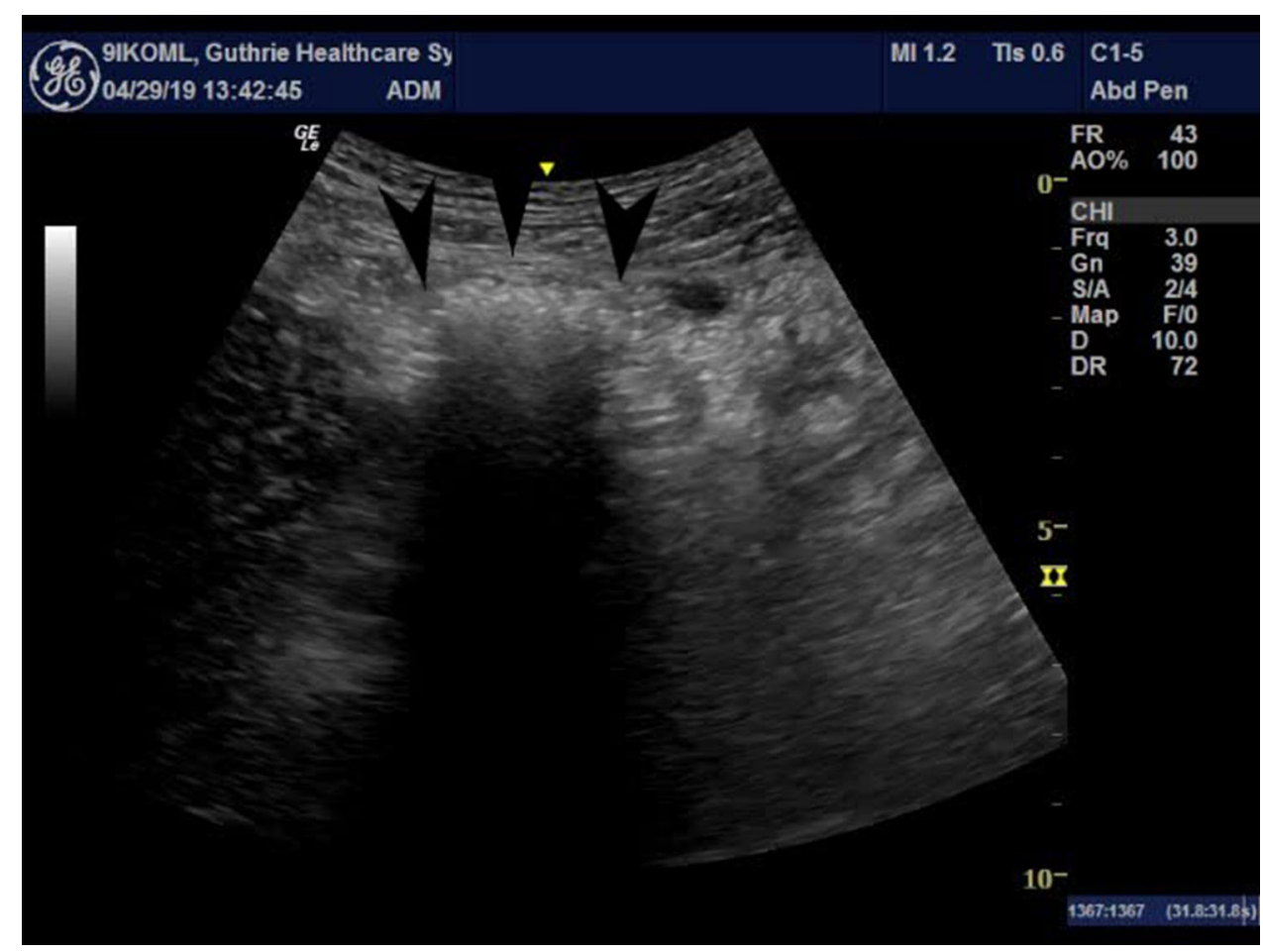

during THR. This approach permits expeditious intraoperative retrieval of the foreign body.

Conflicts of interest None declared.

Editorial responsibility This submission was handled by Dr. Steven Backman, Associate Editor, Canadian Journal of Anesthesia.

Open Access This article is distributed under the terms of the Creative Commons Attribution-NonCommercial 4.0 International License (http://creativecommons.org/licenses/by-nc/4.0/), which permits any noncommercial use, distribution, and reproduction in any medium, provided you give appropriate credit to the original author(s) and the source, provide a link to the Creative Commons license, and indicate if changes were made.

\section{References}

1. Wolford ML, Palso K, Bercovitz A. Hospitalization for total hip replacement among inpatients aged 45 and over: United States, 2000-2010. DCHS Data Brief 2015; 186: 1-8.

2. American Academy of Orthopaedic Surgeons. Projected volume of primary and revision total joint replacement in the U.S. 2030 to 2060. Research News - 2018 Annual Meeting Media Center. Available from URL: https://aaos-annualmeeting-presskit.org/ 2018/research-news/sloan_tjr/ (accessed August 2019).

3. Siddiqi A, Talmo CT, Bono JV. Intraoperative femoral head dislodgement during total hip arthroplasty: a report of four cases. Arthroplast Today 2018; 4: 44-50.

Publisher's Note Springer Nature remains neutral with regard to jurisdictional claims in published maps and institutional affiliations. 